\title{
Potential Mosquito Vectors for Shuni Virus, South Africa, 2014-2018
}

\author{
Milehna Mara Guarido, Thopisang Motlou, Megan A. Riddin, Caitlin Maclntyre, Sontaga Cris Manyana, \\ Todd Johnson, Maarten Schrama, Erin E. Gorsich, Basil D. Brooke, A. Paulo G. Almeida, Marietjie Venter
}

Shuni virus is associated with neurologic and febrile illness in animals and humans. To determine potential vectors, we collected mosquitoes in South Africa and detected the virus in species of the genera Mansonia, Culex, Aedes, and Anopheles. These mosquitoes may be associated with Shuni virus outbreaks in Africa and emergence in other regions.

The genus Orthobunyavirus (family Peribunyaviri1 dae) includes emerging arthropodborne viruses associated with human and animal disease worldwide (1). In 1966, orthobunyavirus Shuni virus (SHUV) was isolated from a cow, Culicoides midges, and a febrile child in Nigeria (2); SHUV recently emerged in Israel, where it has been associated with birth defects in ruminants (3). SHUV has been associated with neurologic disease in horses and wildlife $(4,5)$ and was recently implicated in human cases of neurologic disease in South Africa (6). SHUV was detected in fieldcaught Culex theileri mosquitoes in the 1970s (5), and Culicoides midges have been suggested as vectors (7). We investigated mosquitoes collected in northeastern parts of South Africa to identify their potential as vectors of orthobunyaviruses in the Simbu serogroup of arboviruses, including SHUV.

\section{The Study}

We collected mosquitoes across 5 provinces of South Africa (Figure 1). Site selection was based on his-

Author affiliations: University of Pretoria, Pretoria, South Africa (M.M. Guarido, T. Motlou, M.A. Riddin, C. Maclntyre, S.C. Manyana, T. Johnson, A.P.G. Almeida, M. Venter); Copperbelt University, Kitwe, Zambia (T. Johnson); Leiden University, Leiden, the Netherlands (M. Schrama); University of Warwick, Coventry, UK (E.E. Gorsich); National Institute for Communicable Diseases/NHLS, Johannesburg, South Africa (B.D. Brooke); University of the Witwatersrand, Johannesburg (B.D. Brooke); NOVA University of Lisbon, Lisbon, Portugal (A.P.G. Almeida)

DOI: https://doi.org/10.3201/eid2712.203426 torical outbreaks of arboviruses, including SHUV, in animals $(4,5)$ and humans (6). During January 2014May 2017, we collected mosquitoes monthly; we performed additional collections in 2017 in and around the Kruger National Park (8). In 2018, we performed 1 collection per site during January-May.

We used multiple types of dry ice (carbon dioxide) baited traps: nets, CDC miniature light traps (https://www.johnwhock.com), and BG-Sentinel traps (https://www.bg-sentinel.com). We set traps during 3:30-6:00 PM and emptied them during 5:00-8:00 AM. We killed mosquitoes by freezing and then morphologically identified them to the species level. We pooled females ( $\leq 50$ individuals) by species, collection site, and month. We selected mosquitoes for screening from pools collected during January-June, which represents late summer and autumn, when arbovirus infections in animals and humans in South Africa increase. We obtained climate data from the South African Weather Service (http://www.weathersa.co.za).

For the virus assays, we produced homogenate pools by placing 5 sterile glass beads in microcentrifuge tubes containing $2 \mathrm{~mL}$ of reconstituted minimum essential medium, which we then vigorously shook and clarified. The resulted supernatant was stored at $-80^{\circ} \mathrm{C}$. To extract viral RNA from $200 \mu \mathrm{L}$ homogenate, we used an RNeasy mini kit (QIAGEN, https:/ / www.qiagen.com) according to the manufacturer's instructions. We screened extracted RNA by using 2 PCRs, each targeting the nucleocapsid (NP) gene on the small (S) segment: a Simbu serogroup/orthobunyavirus-specific one-step TaqMan real-time reverse transcription PCR targeting a 152-bp fragment (4) and an SHUV nested real-time RT-PCR targeting a 460bp fragment (9). In an attempt to obtain larger fragments, we performed an SHUV conventional PCR with published primers (10).

For mosquito barcoding (species identification), we extracted DNA from $50 \mu \mathrm{L}$ of the homogenate by using a QIAGEN DNeasy Blood \& Tissue Kit according 


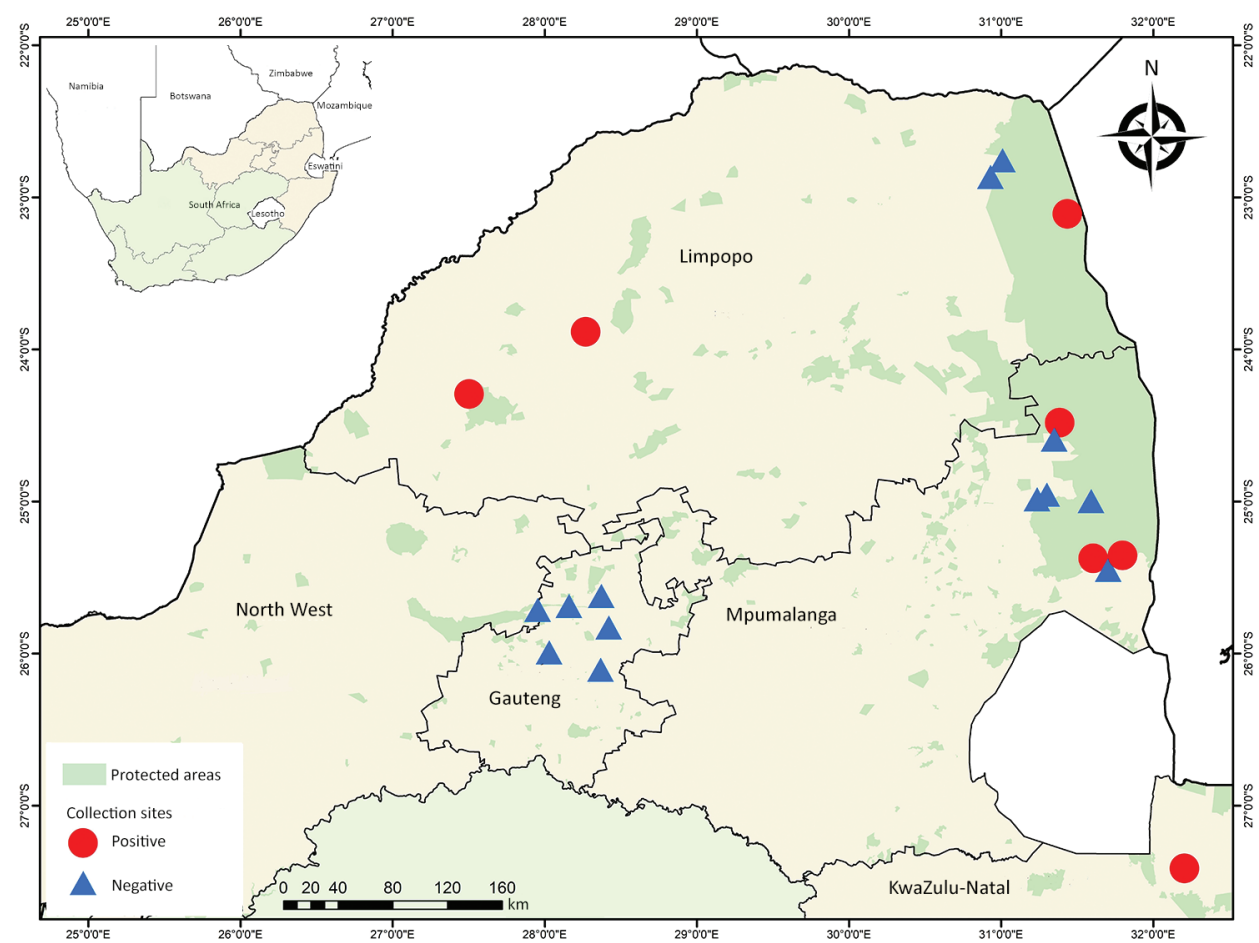

Figure 1. Mosquito collection sites indicating collection locations of Shuni virus-positive (circles) and negative (triangles) mosquito pools, South Africa, January 2014-May 2018. Inset map shows location of South Africa in Africa.

to the manufacturer's instructions. The subunit I of the cytochrome oxidase gene was amplified by using universal primers (11).

All products of the expected size were sequenced by Sanger sequencing at the Forestry Agriculture Bioinformatics Institute, University of Pretoria (Pretoria, South Africa). We compared the resulting sequences by using BLAST (https://blast.ncbi.nlm. nih.gov/Blast.cgi) with sequences available from GenBank, including SHUV strains from South Africa, Nigeria, and Israel and other representative members of Simbu serogroup. For the cytochrome oxidase gene, we selected representative mosquito sequences from GenBank and BOLD (https:// v3.boldsystems.org). We compiled multiple sequence alignments by using MAFFT (https:/ / mafft. cbrc.jp/alignment/software), produced maximumlikelihood trees by using MEGA 7.0 (https://www. megasoftware.net), and calculated maximum-likelihood estimates of mosquito infection rates by using PooledInfRate (https://www.cdc.gov/ncidod/ dvbid/westnile/software.htm).

Of the 64,603 adult mosquitoes collected as described $(8,12)$, we tested 39,035 females. A total of 11 pools were positive for SHUV (Table 1). No other orthobunyaviruses were detected. Positive pools for SHUV were detected in conservation areas $(6 / 11$, $54.5 \%$ ) and rural areas $(5 / 11,45.5 \%)$ (Figure 1). Populations of the SHUV-positive mosquito species peaked with the heavy rains and with the highest mean air temperatures (Appendix Figure 1, https:/ / wwwnc.cdc.gov/EID/article/27/12/20-3426-App1. pdf), which promote establishment of breeding sites and favorable habitats for developing stages and subsequent population growth.

The maximum-likelihood phylogeny based on the genus Orthobunyavirus PCR fragment of 152bp of the S segment showed that all SHUV viruses from the mosquitoes clustered with the Simbu serogroup (Appendix Figures 1,2) and were closest to SHUV on the basis of p-distance analyses (data not shown). For 5 samples, a larger region of the $S$ segment could be amplified to confirm the clustering with SHUV strains previously identified in horses and wildlife from South Africa (Figure 2) and p-distances of $94 \%-100 \%$ with strains previously identified in South Africa, Israel, and Nigeria. Mosquito barcodes consisting of 517-bp were used to build a maximum-likelihood tree (Appendix Table 1, Figures 1-3). The barcoding confirmed all morphologic identifications except for a pool of damaged Aedes spp. mosquitoes and for Ae. subargenteus mosquitoes (for which no other sequence was available in the databases).

Of the 11 pools of SHUV-positive mosquitoes, species belonged to the genera Mansonia (5 pools), Aedes ( 3 pools), Culex ( 2 pools), and Anopheles (1 pool) (Table 2). Previously, SHUV had been detected in 
Table 1. Mosquito species positive for Shuni virus, South Africa, January 2014-May 2018

\begin{tabular}{lccc}
\hline Species & No. assayed & No. pools positive/no. pools tested & Infection rate, $\%\left(95 \%\right.$ Cl) $^{*}$ \\
\hline Anopheles pharoensis & 27 & $1 / 4$ & $39.0(2.4-212.0)$ \\
\hline Culex theileri & 508 & $1 / 22$ & $1.9(0.1-9.0)$ \\
Cx. annulioris & 120 & $1 / 7$ & $6.7(0.5-33.6)$ \\
\hline Mansonia africana & 340 & $3 / 13$ & $8.7(2.6-23.3)$ \\
Ma. uniformis & 2,428 & $2 / 62$ & $0.8(0.1-2.7)$ \\
\hline Aedes subargenteus & 1 & $1 / 1$ & Not applicable† \\
Ae. mcintoshi & 3,653 & $1 / 87$ & $0.3(0.0-1.3)$ \\
Aedes spp. & 273 & $1 / 25$ & $3.6(0.2-17.2)$ \\
\hline Total & 7,350 & $11 / 221$ & \\
\hline${ }^{*}$ Maximum-likelihood estimation: no. positive/no. mosquitoes assayed $\times 1,000$. & & \\
tWhen all pools tested were positive for Shuni virus, the likelihood methods failed. & & \\
\hline
\end{tabular}

Cx. theileri mosquitoes collected in the 1970s near Johannesburg, South Africa (5). In that study, 2 pools of SHUV-positive Cx. theileri mosquitoes were also identified, although mosquitoes of this species were not abundant in the sites detected.

The highest rate of SHUV detection was in Mansonia uniformis mosquitoes, which were found in high numbers at the Shuni virus-positive pool collection

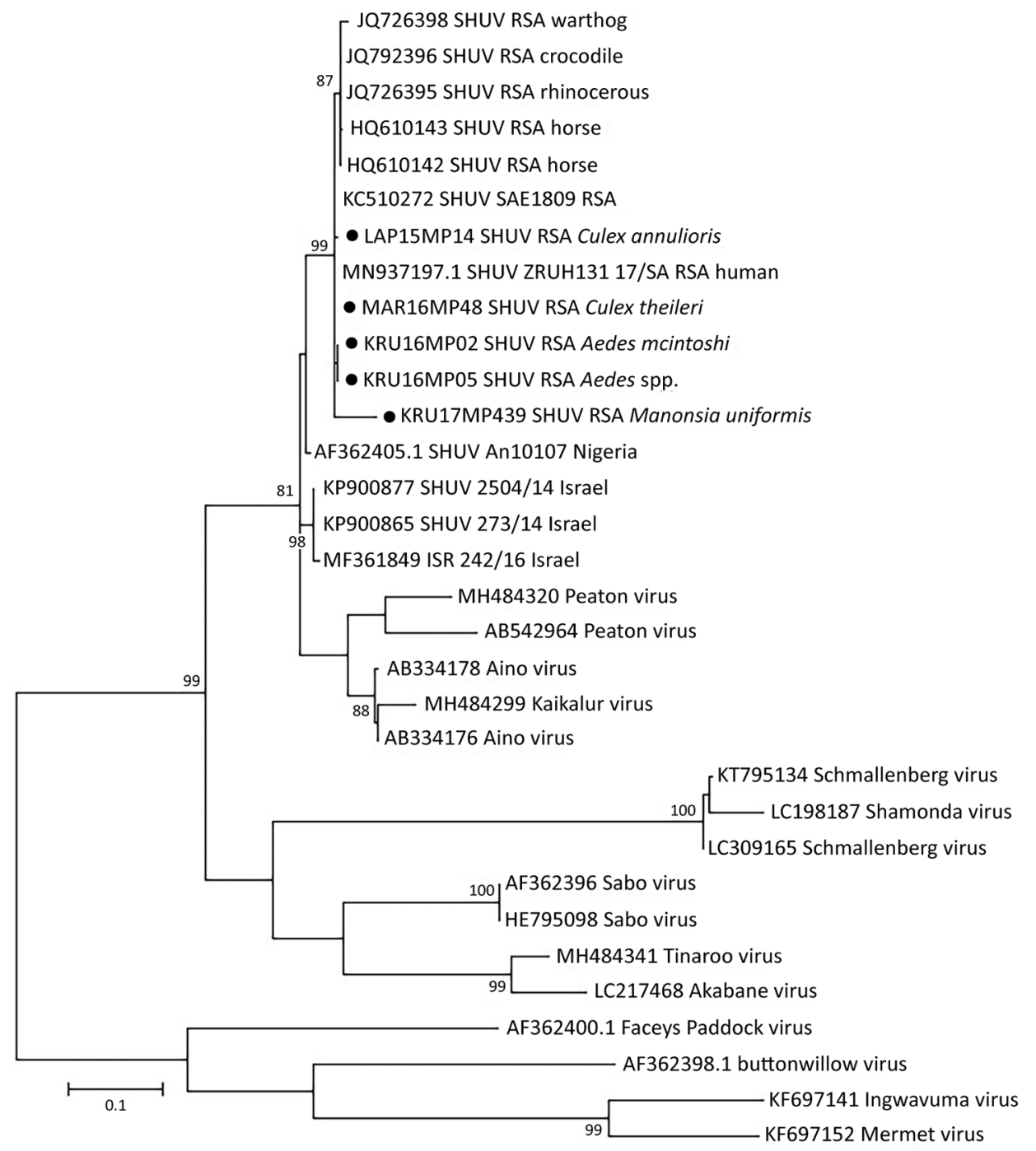

sites. Three other arboviruses have been isolated from M. uniformis mosquitoes in South Africa: Wesselsbron, Ndumu, and Spondeweni (13). M. africana mosquitoes tested positive, but only small numbers of these mosquitoes were collected. Mansonia spp. mosquitoes can feed readily on humans and animals (13) and could have a potential epidemiologic role as bridge species for transmission between animals and humans.

Figure 2. Phylogenetic tree of SHUV-positive homogenate mosquito pools, South Africa, January 2014-May 2018 (black dots), based on 32 sequences and $328 \mathrm{bp}$ of the nucleocapsid gene on the small segment. The tree was constructed with MEGA 7 software (https:// www.megasoftware.net) by using the maximum-likelihood method and the Kimura 2-parameter model with 1,000 bootstrap replicates and includes members of the Simbu serogroup. The tree with the highest log likelihood (-299.13) is shown. GenBank accession numbers are indicated for the new and reference strains, which were selected from SHUV strains identified in South Africa among horses and wildlife $(4,9)$ as well as strains from Nigeria and Israel available in GenBank. Numbers on internal branches indicate bootstrap values. RSA, South Africa; SHUV, Shuni virus. 
Table 2. Mosquitoes positive for Shuni virus, South Africa, January 2014-May 2018*

\begin{tabular}{|c|c|c|c|c|c|}
\hline Species & Site & ID no. & Pool size & $\begin{array}{l}\text { GenBank SHUV } \\
\text { accession no. }\end{array}$ & $\begin{array}{l}\text { GenBank COI } \\
\text { accession no. }\end{array}$ \\
\hline Anopheles. pharoensis & Marakele & Mar16mp59 & 12 & NA & MT428079 \\
\hline Culex theileri & Marakele & Mar16mp48 & 3 & MN914125 & MT428080 \\
\hline Cx. annulioris & Lapalala & Lap15mp15 & 4 & MN914124 & MT428086 \\
\hline Mansonia uniformis & KNP & Knp17mp758 & 9 & NA & MT428087 \\
\hline Ma. africana & KNP & Knp17mp761 & 41 & NA & MT428089 \\
\hline Ma. uniformis & KNP & Knp17mp755 & 2 & NA & MT428088 \\
\hline Ma. africana & KNP & Knp17mp753 & 1 & NA & MT434140 \\
\hline Aedes subargenteus & Jozini & Kzn17mp108 & 2 & NA & MT428082 \\
\hline Ae. mcintoshi & Mnisi & Kru16mp02 & 4 & MT433095 & MT428081 \\
\hline Aedes spp. & Mnisi & Kru16mp05 & 8 & MT433096 & MT428085 \\
\hline Ma. uniformis & Mnisi & Kru17mp439 & 50 & MT433097 & MT428083 \\
\hline
\end{tabular}

Mosquitoes of other species that tested positive included Aedes mcintoshi and Ae. subargenteus. Positive Ae. mcintoshi mosquitoes were collected from Mnisi, where they were the most abundant Aedes spp. at that site. They are considered nonspecific/opportunistic feeders and have a broad range of mammal hosts (14). Ae. subargenteus mosquitoes are tree hole mosquitoes and are either rare in South Africa (14) or are not attracted to the traps used in our study. Although little information about those mosquitoes is available, they might have a strong preference for biting humans (14).

Although SHUV has been detected in mosquitoes, recent studies have also implicated Culicoides spp. midges as potential competent vectors (15). An investigation of the vector competence of Culicoides midges and laboratory-reared $C x$. pipiens and Ae. aegypti mosquitoes for SHUV (7) indicated that neither species of mosquito was susceptible but that Culicoides midges demonstrated the capacity to transmit SHUV. No Ae. aegypti and Cx. pipiens field-caught mosquitoes tested positive for SHUV in this or other studies. Vector competence studies that used SHUVpositive species of mosquitoes identified in our study may define appropriate mosquito vectors and their role in the transmission of SHUV to animals and humans in Africa and the risk to areas where they are found outside the continent.

\section{Conclusions}

Entomologic surveillance for orthobunyaviruses revealed a wide range of potential mosquito vectors for SHUV. We identified SHUV in different species of mosquitoes in South Africa, where cases with neurologic signs have been detected in animals $(4,5)$ and humans (6). The identified mosquito species have also been associated with other arboviruses across Africa. SHUV recently emerged in Israel, where it is associated with neurologic disease and birth defects in animals (3). Mosquitoes of the identified species are potential vectors of SHUV and may be associated with SHUV outbreaks in Africa and further emergence in new regions.

\section{Acknowledgments}

We are grateful to Lapalala Wilderness, Marataba Conservation, South African National Park, for logistical assistance and permission to collect mosquitoes. We acknowledge Leo Braack and Danny Govender for their valuable contribution to mosquito collection. We are grateful to the South Africa Weather Service for providing data for this study.

This study was supported by Cooperative Agreement no. 5 NU2GGH001874-02-00, funded by the Centers for Disease Control and Prevention. It was also funded in part through scholarships from the National Research Foundation and The World Academy of Sciences. A.P.G.A. has been a recipient of the Visiting Professor Programme by the University of Pretoria, South Africa, and acknowledges the Global Health and Tropical Medicine unit. This study was also funded by the Gratama Fund (grant no. 2016.08), the Uyttenboogaart-Eliasen foundation (SUB.2016.12.08), and the RCN-IDEAS travel grant to M.S. and E.E.G.

\section{About the Author}

Dr. Guarido completed her PhD degree and performs postdoctoral work at the Faculty of Veterinary Sciences, University of Pretoria. Her primarily research interest is the study of mosquitoborne viruses, with a focus on the entomology aspects of arbovirus transmission cycles.

\section{References}

1. Hughes HR, Adkins S, Alkhovskiy S, Beer M, Blair C, Calisher $\mathrm{CH}$, et al.; ICTV Report Consortium. ICTV virus taxonomy profile: Peribunyaviridae. J Gen Virol. 2020;101:1-2. https://doi.org/10.1099/jgv.0.001365

2. Causey OR, Kemp GE, Causey CE, Lee VH. Isolations of Simbu-group viruses in Ibadan, Nigeria 1964-69, including 
the new types Sango, Shamonda, Sabo and Shuni. Ann Trop Med Parasitol. 1972;66:357-62. https:/ / doi.org/10.1080/ 00034983.1972.11686835

3. Golender N, Brenner J, Valdman M, Khinich Y, Bumbarov V, Panshin A, et al. Malformations caused by Shuni virus in ruminants, Israel, 2014-2015. Emerg Infect Dis. 2015;21:22678. https://doi.org/10.3201/eid2112.150804

4. Steyn J, Motlou P, van Eeden C, Pretorius M, Stivaktas VI, Williams J, et al. Shuni virus in wildlife and non-equine domestic animals in South Africa. Emerg Infect Dis. 2020;26:1521-5. https://doi.org/10.3201/eid2607.190770

5. van Eeden C, Williams JH, Gerdes TG, van Wilpe E, Viljoen A, Swanepoel R, et al. Shuni virus as cause of neurologic disease in horses. Emerg Infect Dis. 2012;18:318-21. https://doi.org/10.3201/eid1802.111403

6. Motlou T, Venter M. Shuni virus in cases of neurological diseases in humans, South Africa. Emerg Infect Dis. 2020;27:567-9.

7. Möhlmann TWR, Oymans J, Wichgers Schreur PJ, Koenraadt CJM, Kortekaas J, Vogels CBF. Vector competence of biting midges and mosquitoes for Shuni virus. PLoS Negl Trop Dis. 2018;12:e0006993. https://doi.org/10.1371/ journal.pntd.0006993

8. Gorsich EE, Beechler BR, van Bodegom PM, Govender D, Guarido MM, Venter M, et al. A comparative assessment of adult mosquito trapping methods to estimate spatial patterns of abundance and community composition in southern Africa. Parasit Vectors. 2019;12:462. https:/ / doi.org/10.1186/ s13071-019-3733-z

9. Van Eeden C, Zaayman D, Venter M. A sensitive nested real-time RT-PCR for the detection of Shuni virus. J Virol Methods. 2014;195:100-5. https://doi.org/10.1016/ j.jviromet.2013.10.008

10. Bowen MD, Trappier SG, Sanchez AJ, Meyer RF, Goldsmith CS, Zaki SR, et al.; RVF Task Force. A reassortant bunyavirus isolated from acute hemorrhagic fever cases in Kenya and Somalia. Virology. 2001;291:185-90. https:/ / doi.org/10.1006/viro.2001.1201

11. Folmer O, Black M, Hoeh W, Lutz R, Vrijenhoek R. DNA primers for amplification of mitochondrial cytochrome c oxidase subunit I from diverse metazoan invertebrates. Mol Mar Biol Biotechnol. 1994;3:294-9.

12. Guarido MM, Riddin MA, Johnson T, Braack LEO, Schrama M, Gorsich EE, et al. Aedes species (Diptera: Culicidae) ecological and host feeding patterns in the north-eastern parts of South Africa, 2014-2018. Parasit Vectors. 2021;14:339. https:// doi.org/10.1186/s13071-021-04845-9

13. Sharp BL, Appleton CC, Thompson DL, Meenehan G. Anthropophilic mosquitoes at Richards Bay, Natal, and arbovirus antibodies in human residents. Trans R Soc Trop Med Hyg. 1987;81:197-201. https:/ / doi.org/10.1016/ 0035-9203(87)90214-8

14. Muspratt J. The Stegomyia mosquitoes of South Africa and some neighbouring territories. Memoirs of the Entomological Society of Southern Africa. 1956;4:1-138.

15. Snyman J, Venter GJ, Venter M. An investigation of Culicoides (Diptera: Ceratopogonidae) as potential vectors of medically and veterinary important arboviruses in South Africa. Viruses. 2021;13:1978. https:// doi.org/10.3390/v13101978

Address for correspondence: Marietjie Venter, Zoonotic Arboand Respiratory Virus Program, Centre for Viral Zoonosis, Department Medical Virology, Pathology Building, Prinshof Campus South, University of Pretoria, Private Bag X323, Gezina 0031, South Africa; email: marietjie.venter@up.ac.za

\section{EID Podcast \\ A Decade of Fatal Human Eastern Equine Encephalitis Virus Infection, Alabama}

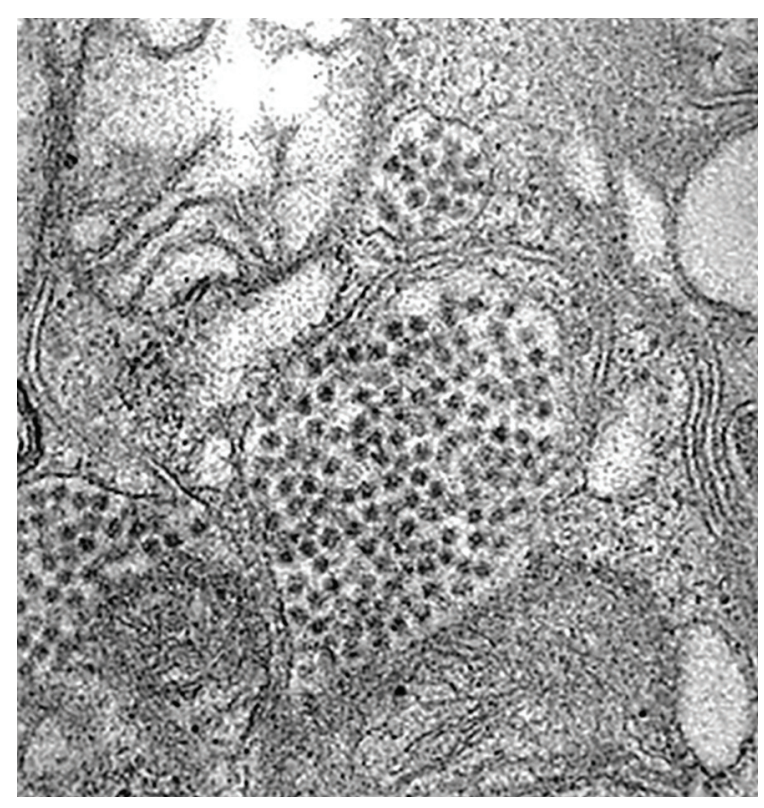

After infection with eastern equine encephalitis virus, the immune system races to clear the pathogen from the body. Because the immune response occurs so quickly, it is difficult to detect viral RNA in serum or cerebrospinal samples.

In immunocompromised patients, the immune response can be decreased or delayed, enabling the virus to continue replicating. This delay gave researchers the rare opportunity to study the genetic sequence of isolated viruses, with some surprising results.

In this EID podcast, Dr. Holly Hughes, a research microbiologist at CDC in Fort Collins, Colorado, describes a fatal case of mosquitoborne disease.

\section{Visit our website to listen: https: //go.usa.gov/xFUhU}

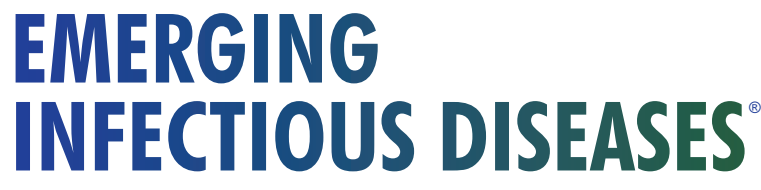

